\title{
Reguetón típico de Marruecos: formas de afirmación política de las niñas a través del baile ${ }^{1}$
}

\author{
Typical reggaeton in Morocco: forms of female political affirmation \\ throught dance
}

\author{
Livia JiMÉNEZ SEDANO \\ Universidade Nova de Lisboa, Portugal \\ liviajs@hotmail.com
}

Recibido: 27/05/2015

Revisado: 08/06/2015

Aceptado: 25/11/2015

Disponible on line: 20/01/2016

\begin{abstract}
Resumen
A través de un relato etnográfico, el texto analiza cómo el baile social puede convertirse en discurso de afirmación cultural de un grupo subordinado. Se describe cómo, frente a un complejo de miradas que construían a un grupo de niñas como marroquíes, musulmanas, poco atractivas, o como objetos de educación e intervención, ellas respondieron afirmando su propia cultura con un discurso corporal inesperado. La forma en que las miradas construyen cuerpos se explora a través de metáforas: una mano que toca, un cincel que esculpe, un látigo que fustiga y una telaraña que controla y atrapa los cuerpos. Debido a esta dimensión política del baile, los talleres también pueden ser una herramienta de opresión y silenciamiento; para evitarlo, se concluye con una serie de recomendaciones para implementar el baile en los procesos de intervención social.
\end{abstract}

Palabras clave: baile social, cuerpo político, miradas, Islam, etnicidad.

\begin{abstract}
Through an ethnographic account, this text analyses how social dance may become a discourse involving the cultural affirmation of a subordinate group. It describes how a group of girls faced with a complex of outlooks that construed them as Moroccan, Muslim or unattractive —or as objects of education and intervention — responded by affirming their own culture with an unanticipated corporal discourse. The way in which looking construes bodies is explored through metaphors: a hand that touches, a chisel that sculpts, a whip that lashes and a cobweb that controls and traps bodies. Owing to this political dimension of dance, workshops can also be an oppressive and silencing tool; to prevent this, the article concludes with a series of recommendations to implement dance in social intervention processes.
\end{abstract}

Keywords: social dance, political body, outlooks, Islam, ethnicity.

Referencia normalizada: Jiménez Sedano, L. (2015): «Reguetón típico de Marruecos: formas de afirmación política de las niñas a través del baile». Cuadernos de Trabajo Social, 29(1): 73-81.

Sumario: Introducción. 1. «iLas niñas están bailando!»: auto-afirmación en movimiento. 2. El reguetón típico de Marruecos: la subversión de mostrar la propia cultura. 3. Un cuerpo esculpido con el cincel las miradas. 4. La telaraña de miradas como forma de control social. 5. La conflictividad del cuerpo musulmán femenino danzante. 6. Reflexión final: el baile y la intervención social. 7. Referencias bibliográficas.

\section{Introducción}

El arte se ha revelado como una de las herramientas más interesantes en los procesos de in- tervención social (Moreno González, Usán, Criado y Santaforentina, 2013). En el contexto de la escuela, los programas que plantean desde

1 Una versión preliminar de este texto fue presentada oralmente en las Jornadas «Las Otras: Experiencias de mujeres en un mundo interconectado», celebrado en Sevilla en septiembre de 2014 y organizado por el Grupo de Investigación Escritoras y Escrituras de la Universidad de Sevilla. Gracias a la generosa invitación a este evento de Olga Torres y Mercedes Arriaga surgieron estas reflexiones. 
la perspectiva del Trabajo Social para mejorar la convivencia tienen que partir del reconocimiento $\mathrm{y}$ aprovechamiento de la diversidad cultural existente (García, 2009). Sin embargo, según como se trabaje, se pueden conseguir efectos contrarios a los deseados, ya que el encuentro entre culturas nunca se realiza en términos de igualdad (Vázquez, 2001): por ejemplo, las actividades interculturales pueden convertirse en ejercicios de intercambio folclórico que refuerzan los estereotipos en lugar de contribuir a combatirlos (Carbonell i Paris, 1998). El objetivo de este texto es aportar elementos de reflexión para trabajar con el baile en los procesos de intervención social. Con una mirada antropológica, se narran episodios de transformación social que acontecieron a través del baile. A partir de ello, propongo una explicación y concluyo con recomendaciones concretas para emplear la danza en los proyectos de intervención social.

Este trabajo parte de la tesis doctoral en Antropología que defendí en 2011. El objetivo era explorar de qué formas los niños y las niñas construyen pertenencias étnicas en contextos multiétnicos. Entre 2002 y 2007 realicé el trabajo de campo, comparando dos contextos, uno en Madrid y otro en Andalucía: observación participante en la escuela, actividades extraescolares, el parque, el hogar, las visitas a familiares y amigos, las fiestas domésticas, los cultos religiosos y las asociaciones, complementado con entrevistas en profundidad y análisis de documentos. Entre 2004 y 2005, con una beca de la Universidad de Granada, participé en la evaluación del Plan de atención educativa al alumnado inmigrante (PAEI) de la Junta de Andalucía, que llevaba a cabo el entonces Laboratorio de Estudios Interculturales. Uno de los centros presentaba en sus estadísticas un 50 por ciento de marroquies $^{2}$, y decidimos que teníamos que acudir allí. El barrio era tan fascinante que los dos meses iniciales de etnografía se convirtieron en dos años. Entre 2005 y 2007 visité casi a diario el barrio, que llamaremos Los Churumbeles ${ }^{3}$, por la gran cantidad de niños y niñas que animaban sus calles. En 2007 fui contratada como antropóloga para acompañar el proyecto europeo Equal-Atenea en Los Churumbeles. El objetivo era realizar una investigación antropológica aplicada que pudiera ser útil para los profesionales de la intervención social que trabajaban en el barrio.

La Junta de Andalucía lo había catalogado como ZNTS (Zona con Necesidades de Transformación Social), lo que significaba que acumulaba problemas como altos índices de desempleo, absentismo escolar o consumo de drogas, y era zona de actuación prioritaria de la Consejería para la Igualdad y Bienestar Social. Los Churumbeles acabó siendo uno de los primeros destinos de los trabajadores que llegaban de Marruecos. Aunque las viviendas pertenecían oficialmente a la Junta de Andalucía, se había instalado un mercado inmobiliario informal muy lucrativo. Como resultado de ello, en apenas cinco años el porcentaje de habitantes de origen marroquí pasó del 0 al 50 por ciento. La Empresa Pública del Suelo Andaluz (EPSA) llegó al barrio con la intención de regularizar la situación de las viviendas, para luego reconstruir la zona que estaba en peor estado. Lo primero que hicieron fue un registro de los habitantes de hecho, arrojando las siguientes estadísticas: el 45 por ciento marroquíes, el 54 por ciento españoles y el 1 por ciento de otras nacionalidades. Entre los españoles, contabilizaron el 24 por ciento gitanos y el 30 por ciento castellanos ${ }^{4}$. Estos marroquíes venían de regiones muy diversas, tanto urbanas como rurales.

Durante estos años descubrí algo que no esperaba: el baile era uno de los elementos más importantes para crear dinámicas de identificación y distinción en la vida cotidiana. Por casualidad acabé ejerciendo de maestra de baile (danza oriental y fusión flamenco-árabe), y comencé a observar el movimiento de los cuerpos de forma más detenida. Llegué a la conclusión de que era necesario profundizar en las relaciones entre cuerpo, baile y etnicidad, y así elaboré el proyecto postdoctoral en el que trabajo actualmente: «Etnicidades danzantes en un mundo social transnacional» $\rangle^{5}$. Entre 2012 y 2014 participé en el proyecto $\mathrm{I}+\mathrm{D}$ «Cultura, Género e Islamofobia:

${ }^{2}$ Las expresiones emic (nativas) aparecen en cursiva en el texto. Considero los etnónimos categorías emic (Díaz de Rada y Jiménez Sedano, 2011), y por lo tanto aparecen en cursiva.

${ }^{3}$ Los nombres de lugares concretos y personas son ficticios para proteger el anonimato de los informantes.

${ }^{4}$ Eufemismo local para decir español no gitano.

${ }^{5}$ Financiado por la Fundação para a Ciência e Tecnologia (gobierno de Portugal). 
El Islam en la Diaspora», coordinado por Ángeles Ramírez. Este trabajo en torno a los contextos transnacionales actuales de las prácticas del Islam me ayudó a replantear y contextualizar mejor lo que había observado en el barrio.

Desde la perspectiva de la antropología de la danza y gracias a los enriquecedores intercambios con el equipo $\mathrm{I}+\mathrm{D}$, algunas observaciones cobraron nueva luz. Lo que parecían anécdotas de campo resultan ahora episodios reveladores que nos pueden dar claves para entender mejor el significado social del baile y su potencial para la transformación social en contextos concretos. Lo que parecían anécdotas de campo resultan ahora episodios reveladores que nos pueden dar claves para entender mejor el significado social del baile y su potencial para la transformación social en contextos concretos.

\section{1. «iLas niñas están bailando!»: auto-afirma- ción en movimiento}

En la escuela había conflictos entre el alumnado obediente a las maestras y el bando más rebelde, que en los últimos años era el más numeroso y había conseguido un amplio poder de decisión sobre la vida del centro escolar (Jiménez Sedano, 2012). A los recién llegados, que no se unían a este movimiento de insumisión escolar, se les llamaba moros y eran víctimas de insultos y ataques, con frecuentes peleas en el patio. En ese contexto, Manuel, el maestro de ATAL ${ }^{6}$, pensó en una estrategia para mostrar a su alumnado un mundo social menos conflictivo: un intercambio cultural con otro centro donde había trabajado anteriormente, que llamaremos IES La Sierra. Ese día el profesorado organizaba talleres lúdicos y el alumnado protagonizaba espectáculos de música y baile. Según el guión académico, se trataba de un intercambio entre la cultura española y la cultura marroquí. El primer año en que se organizó fue en 2003, y participé por primera vez en 2005.

Fue allí donde adquirí el rol de maestra de baile. Todas las maestras colaboraban de alguna forma, me invitaron a acudir y me sugirieron que ofreciera algún taller. Era una buena oportunidad para corresponder con reciprocidad a la ayuda que me estaban brindando, abriéndome las puertas y concediéndome entrevistas. No tengo una formación sólida en danza ${ }^{7}$ y en aquel momento no tenía ningún objetivo pedagógico ni artístico; se trataba más bien de una estrategia etnográfica para adquirir un rol reconocible y apropiado en el campo. Llevaba varios años aprendiendo danza oriental como afición y preparé un taller sencillo para que las niñas se divirtieran.

El 23 de febrero de 2005 se celebró el intercambio cultural en el IES La Sierra. Dos compañeras de la universidad de Granada, Toñi Olmos y Yanet Lorenzo, acudieron con nosotras y estuvieron ayudando en la organización, así como observando y grabando el encuentro. En el taller de danza oriental aparecieron treinta alumnas. Así lo describí en el diario de campo:

Las niñas marroquíes ${ }^{8}(\ldots)$ ocupan la mayor parte de las primeras filas. Algunas de ellas llevan pañuelo y chilaba. Comienzan las explicaciones de los movimientos. Las niñas marroquíes los siguen bastante bien y me sonríen mucho; las niñas españolas algunas lo hacen mejor que otras. A veces me meto por las filas de atrás para ver si lo están siguiendo bien. Hay dos grupos de niñas (todas españolas) que me dice que sí les sale, pero no quieren hacerlo delante de mí. Se agarran entre ellas y se ríen. Otras me dicen que no les sale, pero que da igual, que no me preocupe. Les da vergüenza. Cuando repiten los movimientos les voy diciendo cuándo les sale bien. Las niñas marroquíes reciben mucho refuerzo porque todo les sale bastante bien. Entre las niñas españolas, algunas sonríen y la mayoría no sonríen tanto, y están más concentradas en tratar de repetir el movimiento.

En los momentos que no les estoy dando instrucciones (por ejemplo, cuando estoy buscando la música) la mayoría de las niñas están paradas, pero las niñas marroquíes empiezan a bailar entre ellas. En un momento de la clase, una de las niñas marroquíes me dice que ellas bailan esa misma canción de otra forma, que si puedo ponerla para

${ }^{6}$ Aulas temporales de adaptación lingüística, terminología propia de la Junta de Andalucía para el programa de clases intensivas de castellano para alumnos extranjeros durante, al menos en teoría, un primera fase de adaptación.

${ }^{7}$ Respeto profundamente el arte y la profesión de la danza. Lo realicé de forma voluntaria, sin cobrar, y no había profesionales de la danza en el campo a los que hiciera competencia desleal.

${ }^{8}$ En este momento de la tesis aún no había hecho la reflexión crítica sobre los etnónimos y por eso aparecen como categorías descriptivas y sin cursiva. 
que la bailen libremente. Le digo que es muy buena idea, que dentro de un rato lo haré así. Cuando una de las maestras del IES me dice que ya debe quedar poco para el final, les digo que se pongan en círculo y que quiero que vayan saliendo al centro y bailando como ellas quieran. Las que están fuera, en el círculo, deben hacer lo mismo que la que está en el centro. Salen primero las niñas marroquíes, pero no una sino dos juntas, y luego otras dos, y otras dos. Salen en parejas. Al final se ponen todas en el centro y el círculo queda compuesto por niñas españolas. (...) Me voy acercando a ellas para animarlas a salir. Unas dicen que no, otras salen corriendo cuando ven que me acerco. Después de bailar varias canciones, las niñas españolas van saliendo y se van yendo solas. Después las demás (Diario de campo, 23/02/2005).

Después de los talleres, había un partido de fútbol y varias actuaciones programadas; la mayoría, de chicas y chicos del instituto anfitrión mostraban coreografías que habían preparado ellas mismas con temas de moda de reguetón y pop. También había una banda de música que dirigía el profesor de música del IES La Sierra. El profesor de música del IES Los Churumbeles había ensayado con los chavales (todos chicos) un espectáculo de instrumentos variados de percusión. En general en ambos institutos, los chicos tocaban y las chicas bailaban. Las chicas de Los Churumbeles habían traído taqsitas $^{9}$ de sus madres para hacer un pase de modelos. Todo iba transcurriendo según el guión previsto hasta que ocurrió algo inesperado. Volvamos al diario de campo:

Al poco, una de las niñas, Fátima, va a pedir al director del instituto anfitrión que ponga un cassette que ha traído, que ellas también quieren bailar. Salen las niñas que estaban en el taller, con los taqsitas de sus madres, y se ponen a bailar y a cantar en círculo, con la música de una cinta que han puesto. Detrás del público, veo a los profesores que han venido, entre ellos al ATAL, emocionados. Manuel, el ATAL está llorando. Dice que es increíble lo que ha ocurrido, con todo lo que han trabajado con ellas porque son tímidas y tienen miedo en el nuevo contexto y ahora, de pronto, salen en público y cantan y bailan con orgullo (Diario de campo, 23/02/2005).

La estructura del espacio que adoptaron era similar al que pude ver que reproducían en las fiestas de las bodas: un círculo donde miraban al centro y a los lados, se agarraban de las manos, en parejas o grupos de tres, y reían, bromeaban y entonaban juntas la canción. Los movimientos circulares y vibratorios de caderas y hombros eran acompañados con percusión de pies. No se trataba de movimientos de danza oriental de academia ni de música de Egipto, aunque hay una cierta familiaridad kinésica; se trataba de lo que las informantes llaman chaabi ${ }^{10}$, música popular urbana para bailar, de gran éxito en Marruecos. En los talleres que impartí en el Instituto, las niñas exigían que las clases fueran a puerta cerrada con llave y que los chicos no tuvieran permiso para entrar. Todas las maestras estaban admiradas al verlas exhibiéndose, sonriendo y ocupando con aplomo el escenario. Sus compañeros del barrio se llevaban las manos a la cabeza y abrían los ojos con incredulidad, riendo e intercambiando codazos y guiños cómplices. Algunos sacaron sus teléfonos móviles para grabarlas. Pasado el momento de primer impacto, se quedaron en silencio observando atentamente hasta romper en aplausos al final.

\section{El reguetón típico de Marruecos: la sub- versión de mostrar la propia cultura}

$\mathrm{Al}$ curso siguiente, las niñas me pidieron que les diera clases regulares y acepté, pensando que era un óptimo rol de campo. Las más mayores ya habían terminado la secundaria, y el grupo estaba formado por la siguiente generación. Para motivarlas a preparar una actuación, de cara al siguiente encuentro en La Sierra, les puse el DVD del año anterior, 2005. Pensé que querrían hacer algo similar al grupo de niñas del curso pasado, pero para mi sorpresa, no fue exactamente así. La escena aparece relatada en el diario de campo:

Cuando termina el DVD les pregunto qué quieren hacer y Sherezade dice «pero nosotras también tenemos que preparar algo, mira cuántas cosas hicieron los otros». Me dice Rania «iQue no sea todo egipcio, hagamos algo marroquí!» y Zahara y Fátima coinciden «iy una española también!» (...) Luego les pongo La Tarara (fusión de una canción popular marroquí y una canción popular española), pensando que sería una buena opción para canción española y ya tengo, la coreo. La pongo y la bailamos un poco (...) Pero no les

\footnotetext{
9 Vestidos de fiesta.

10 Significa «popular».
} 
gusta. Fátima dice «no, maestra, pero con árabe no, que sea española». Me sorprende, porque está mezclada con una canción popular marroquí y suele gustarles a mis amigos marroquíes (...). Pero ahí está el error de identificar cultura con pasaporte. (...) Fátima dice « ¿Con la de la gasolina (tema popular de reguetón)! ¡Ésa está muy bien, podemos hacer una con la gasolina!». Me quedo descolocada, y más cuando veo la reacción de las demás, Sherezade, Rania... «iSí, sí, la gasolina está muy bien! (Diario de campo, 20/01/2006).

Así comenzaron los ensayos con el objetivo de actuar en La Sierra. Las niñas querían conocer a las chicas, y muy especialmente a los chicos, del otro Instituto. El acto intercultural organizado por el Instituto era así apropiado para otros fines. Las semanas antes de la actuación, todo eran nervios y preparativos: la ropa, el maquillaje, la eterna pregunta «¿Quién nos va a ver?». Nadie hablaba de marroquies ni de españoles. Acabé tan envuelta en su perspectiva del evento que olvidé por completo las expectativas y objetivos de las maestras que lo habían organizado.

El día del intercambio llegó y se sucedieron todas las actividades. Cuando las niñas terminaron de bailar, en mitad de los aplausos se acercó a mí un maestro del IES La Sierra, de los que más se habían implicado en la organización del evento. Me dijo en un tono de broma que no ocultaba un cierto reproche: «Ah, muy bien maestra, «La gasolina», muy típico de Marruecos...» Entonces caí en la cuenta: había olvidado por completo que aquello debía ser un intercambio de culturas reificadas bajo las etiquetas española y marroquí para que el ritual tuviera sentido para las maestras organizadoras. En vez de ello, las niñas habían hecho algo subversivo: mostrar su propia cultura.

\section{Un cuerpo esculpido con el cincel las miradas}

Para las niñas la negociación de las miradas era fundamental: exigían estar protegidas bajo llave de las miradas masculinas durante las clases de baile, su mayor preocupación en los ensayos era saber quién las iba a ver, y todos los problemas en torno al baile no tenían que ver con el movimiento en sí, sino con los observadores del mismo. Muchas maestras encontraban excesivo este celo, pero las miradas no son inocentes ni carecen de efectos sociales, o lo que en Antropología Social se denomina eficacia simbólica (Velasco, 1988). Son como manos que tocan y modifican efectivamente el cuerpo observado. Le Breton (2007) habla en este sentido de la tactilidad de la mirada:

Colocar la mirada sobre el otro nunca fue un acontecimiento anodino; en efecto, la mirada se aferra, se apodera de algo para bien o para mal, es inmaterial sin duda, pero actúa simbólicamente. En ciertas condiciones oculta un temible poder de metamorfosis. No carece de incidencia física para quien de pronto se ve cautivo de una mirada insistente, que lo modifica físicamente: se acelera la respiración, el corazón late con más velocidad, la tensión arterial se eleva, sube la tensión psicológica. (...) La mirada es un contacto: toca al otro y la tactilidad que reviste está lejos de pasar desapercibida en el imaginario social. (...) Sería larga la enumeración de los calificativos que le otorgan a la mirada una tactilidad que hace de ella, según las circunstancias, un arma o una caricia que apunta al hombre en lo más íntimo y en lo más vulnerable de sí mismo (p. 58).

Por ello, en todas las sociedades existe un código implícito que regula las miradas, sus contextos, tiempos y formas apropiados, que se va aprendiendo a lo largo del proceso de socialización; por ejemplo, la norma propiamente urbana de no fijar la mirada en el viandante desconocido o «indiferencia cortés» (Delgado, 1999). Las miradas construyen el espacio: distinguen un entorno más rural (miradas fijas y sostenidas) de otro más urbano (miradas fugaces), y se convierte en criterio para calibrar el nivel de peligrosidad de los barrios en una ciudad. Muchas personas decían sentirse inseguras en Los Churumbeles porque, contaban, «allí todo el mundo se te queda mirando»».

Sin embargo, la mirada (un cierto tipo de mirada) es obligatoria para mostrar reconocimiento e interés en ciertas situaciones sociales (Le Breton, 2007), y el negarla o reducirla se interpreta como hostilidad y falta de educación. Pero esto mismo puede ser experimentado como invasión o agresión al cambiar de contexto cultural (Hall y Hall, 2010). Este extrañamiento cultural es la mejor demostración de que el código, aunque implícito, es profundamente eficaz.

En cualquier caso, eran las niñas las que intentaban protegerse de las miradas de los chicos, y no viceversa: las miradas tienen género y construyen género (Van Nieuwkerk, 2001). Son las miradas masculinas las que subordinan a las mujeres a unos criterios de valor establecidos principalmente sobre su cuerpo (Le Breton, 2007). 
En esta tensión, los hombres escenifican su virilidad a través de la práctica de mirar a las viandantes y las mujeres miden su feminidad en función de su capacidad para atraer miradas masculinas. Las partes del cuerpo más observadas y las formas de mirarlas producen un efecto de conciencia corporal en las observadas y de experiencia de su cuerpo como sexuado y femenino: el cuerpo es socialmente esculpido con el cincel de las miradas. Existe una gran diversidad cultural en las formas de cincelar el cuerpo de las mujeres, como muestran Hall y Hall (2010) en el siguiente ejemplo:

Muchas norteamericanas se sienten muy turbadas al visitar Francia o Italia, debido a que, por primera vez en su vida, los hombres las miran realmente - sus ojos, pelo, nariz, labios, pecho, caderas, piernas, muslos, rodillas, tobillos, pies, vestido, peinado, hasta su forma de andar- Cuando esas mismas mujeres, una vez que se han acostumbrado a que las miren así, regresan a los Estados Unidos, a menudo les asalta el sentimiento de que «ya nadie me mira de verdad» (p. 181).

Para nuestros protagonistas, el juego masculino de invadir con las miradas y el femenino de protegerse estratégicamente de ellas eran fundamentales. Los chicos desarrollaron una performance de sorpresa al ver a sus compañeras bailando en público, las grabaron con el móvil y no les quitaron los ojos de encima: independientemente de su nivel de interés en el espectáculo en sí, estaban actuando conforme a las expectativas culturales de masculinidad. Ellos debían mirar y ellas debían ser miradas para construir recíprocamente el género de ambos. Pero este juego escondía dimensiones menos amables.

\section{La telaraña de miradas como forma de control social}

La eficacia simbólica de las miradas es tal que se convierten en una de las principales herramientas de control social: el miedo a las miradas sostenidas más tiempo del culturalmente debido y la vergüenza ante las miradas de los otros son inculcadas desde la más tierna infancia para controlar a los sujetos sociales. En este sentido, la mirada es como un látigo que golpea al que viola la norma, construyendo el cuerpo observado como un cuerpo político a vigilar y castigar (Foucault, 1986). El conjunto de miradas colectivas teje una telaraña invisible en la que quedan presas aquellas que osan presentar públicamente sus cuerpos de una forma no reglada. En Los Churumbeles, el espacio urbano estaba conformado por una densa telaraña de miradas que nuestras protagonistas sentían como asfixiante en comparación con aquella a la que estaban acostumbradas en Marruecos.

Primero, había vecinas y vecinos que sentía un profundo extrañamiento ante aquellas formas de vestir y cubrir su cuerpo: cada vez que salían a la calle, las observaban fijamente con recelo y desaprobación. Sus cuerpos cubiertos eran el máximo símbolo visible de la vertiginosa transformación social del barrio y lo que era vivido como la «invasión» de un extraño. Algunas compañeras del Instituto se reían abiertamente de su forma de vestir, que construían como poco moderna y ridícula. Eran miradas de desprecio y superioridad.

Segundo, las maestras y profesionales de la intervención social tenían una actitud de mayor respeto a la diferencia, pero con una cierta condescendencia protectora. Temían que las chicas no pudieran completar sus estudios y encontrar un trabajo a causa de tradiciones culturales que las encerrarían en el hogar. Las formas de exhibir modestia en el vestir, hablar, mirar y moverse, se interpretaban como signos de peligro en este sentido. Eran miradas de lástima y preocupación que las construían como objetos de intervención.

Tercero, las miradas más peligrosas. En el barrio se había producido una concentración de personas procedentes de zonas diversas de Marruecos con culturas visuales y de vestimenta muy variadas. A ello se unía el discurso del Islam neoconservador que llegaba a las pantallas de las televisiones de los salones de Los Churumbeles por vía de canales, como Iqra y $\mathrm{Al}-\mathrm{Ja}$ zeera (Haenni, 2005; Gómez, 2009) gracias al bosque de antenas parabólicas que poblaba los tejados del barrio. Y también las tendencias conservadoras más clásicas que traía el imam de la mezquita en el discurso del viernes - una vez dijo que el espacio mixto de estudio de una asociación del barrio era un sitio para ligar para las chicas musulmanas - y al día siguiente, los padres les prohibieron acudir. Estos discursos se basaban en el miedo a la influencia de una cultura no musulmana percibida como extraña y amenazante (Mernissi, 1992). Tanto las chicas como sus familias temían especialmente las miradas de 
la vecindad musulmana más conservadora, que podían extender rumores sobre su falta de vergüenza y arruinar su reputación de cara a un posible matrimonio. Por ello, recibían presiones familiares para cubrirse más de lo que era habitual y deseable para muchas de ellas. Según me contaban, intentaban salir del barrio siempre que podían para pasear por otras zonas de la ciudad, lejos de aquella madeja de miradas en la que sus cuerpos se podían ver atrapados y condenados a morir socialmente en la soltería (Ramírez, 1998). Los hermanos se habían convertido en el agente principal de control y algunos ejercían su autoridad con castigos corporales para que se cambiaran de ropa o no frecuentaran espacios donde podían ser observadas con sospecha.

Por último, estaban las miradas procuradas, deseadas, aquellas que las convertían en mujeres atractivas, aquellas que colmaban sus expectativas culturales y les permitían construir una feminidad socialmente prestigiosa y valorada. La vergüenza convivía contradictoriamente con el deseo de ser observadas. En este contexto, las miradas eran tan imprescindibles, como su prohibición, para que el resultado fuera eficaz simbólicamente: un cuerpo femenino reconocido como tal.

En el taller de danza del intercambio, las niñas se encontraron con un nuevo tipo de miradas: las de admiración de las alumnas del Instituto anfitrión, que no eran capaces de moverse como ellas. Aunque la danza oriental de academia no se correspondía con su propio lenguaje kinésico, era lo bastante semejante a su registro de baile de fiesta chaabi como para hacer una sencilla adaptación y conseguir una performance bastante aceptable. La figura de autoridad en esa situación, la maestra, construyó un escenario social en el cual sus cuerpos en movimiento eran los más valorados en ese campo. En esta encrucijada de miradas, eran precisamente los cuerpos danzantes los que activaban y exacerbaban todo este entramado complejo de expectativas sociales y conflictos.

\section{La conflictividad del cuerpo musulmán fe- menino danzante}

Según Hanna (1988), la forma del movimiento culturalmente pautada en el baile construye el cuerpo como femenino. En el contexto cultural específicamente arabo-musulmán, según Sabbah (1984), las relaciones entre géneros están fuertemente erotizadas, y los cuerpos femeninos se consideran sexuales e incitantes, por definición, ocultando sus dimensiones económica y política; por ello, la ocupación femenina de los espacios públicos se vive como una agresión erótica. El máximo exponente de esta «agresión» es afirmar la presencia del cuerpo a través del movimiento. Según Van Nieuwkerk (2001), investigadora de las bailarinas profesionales en Egipto:

La transgresión más grave de las bailarinas es que se mueven. (...) Moverse es inmoral para las mujeres porque hace llamar aún más la atención sobre sus vergonzosos cuerpos. Cuando pregunté a un shay $k h^{11}$ si las bailarinas de folclore (que llevan más ropas que las bailarinas de oriental) son menos haram $^{12}$, él respondió con resolución: «no, también se mueven (p. 141).

En situación migratoria, los cuerpos femeninos se construyen como los principales símbolos públicos del Islam, lo cual les convierte en los principales objetos de reacciones islamófobas (Mijares, 2006; Téllez, 2008; Ramírez, 2011; Aixelá, 2012). En Los Churumbeles, los liminales cuerpos adolescentes de nuestras protagonistas, en pleno proceso de redefinición social, se habían convertido en el material preferente para simbolizar los múltiples conflictos sociales del barrio. Sus cuerpos se habían hecho muy visibles y sobre ellos se ejercían múltiples presiones culturales contrapuestas. En esta coyuntura, el baile se convirtió para ellas en una herramienta política de primer orden: un jaque corporal a las miradas en el que afirmaban el lugar que ellas querían ocupar en el campo social. Frente a todos los discursos proyectados sobre ellas y vehiculados a través de su corporalidad, sus cuerpos se afirmaron presentes y desgranaron su propio discurso. No estaban reproduciendo la cultura de sus familias ni la de sus compañeros/as de escuela ni obedecían a las expectativas de las maestras: en una tensión entre producción y reproducción (Willis, 2008) estaban en ese momento construyendo su propia cultura, a su manera, y en base a todos los referentes con los que se sentían efectivamente

11 Líder religioso o político considerado sabio.

12 Prohibido por la religión. 
identificadas (incluido el reguetón que escuchaban en casa a diario).

Lo más interesante aquí es que no se trata sólo de una performance aislada e independiente de lo que ocurre en el día a día. El poder transformador del baile radica en que no está separado de las demás esferas de kinésica cotidiana, sino que existe una continuidad con las mismas (Williams, 2004). Forma parte integrante del habitus (Bourdieu, 1987), ese conjunto de registros que se incorporan durante los procesos de socialización. El baile puede entenderse como una musicalización, una estilización de la kinésica cotidiana. No rompe con la corporalidad cotidiana, sino que parte de ella y la celebra, junto con todos los significados culturales asociados. También permite una negociación de roles sociales precisamente porque se basa en ellos.

El proceso de agencia comienza en el momento en que deciden pedir permiso para romper la dinámica de las relaciones kinésicas de poder: querían bailar libremente, según su propio registro ya in-corporado, en lugar de esforzarse por mimetizarse con el modelo externo de la maestra. En ese momento, quedaron convertidas en las únicas autoridades de un tipo de performance, el chaabi, que sólo ellas dominaban. En este nuevo contexto, en lugar de ser observadas por la figura de autoridad escolar a través de la teoría del déficit (por los errores al hablar castellano, por las faltas de ortografía en árabe, por lo que aún no sabían, por lo que aún tenían que aprender), pasaron a ser valoradas por lo que sí tenían, sí sabían, en un escenario compuesto por sujetos sociales que apreciaban este conocimiento. Al independizarse de las instrucciones de la maestra de baile, la agencia sobre el movimiento las convertía en protagonistas de un discurso de movimiento a través del cual transmitían su propio mensaje $y$, al contrario de lo que ocurría habitualmente, en este contexto festivo donde las jerarquías cotidianas quedaban temporalmente suspendidas, fueron escuchadas con atención.

\section{Reflexión final: el baile y la intervención social}

A partir de estas reflexiones sobre las experiencias de campo, propongo una serie de ideas básicas para la utilización del baile en programas de intervención. Debido a la dimensión política que tiene la danza, un taller de baile se puede convertir tanto en una herramienta de opresión y domesticación del cuerpo (Velasco, 2007; Busto, 2012) como en una vía para apoyar formas de afirmación, en un altavoz de los discursos políticos silenciados (Quintero, 2009; Browning, 1995). Según y cómo se plantee y trabaje, podemos obtener resultados muy diferentes y hasta contrapuestos. Por ello, concluyo realizando las recomendaciones siguientes:

1) Conocer el contexto concreto en que se va a trabajar, ello implica escuchar no sólo las voces de maestras y profesionales de la intervención social y saber el trabajo que vienen desarrollando hasta nuestra llegada al terreno, sino también y muy atentamente a las personas protagonistas, así como qué estrategias despliegan para posicionarse en su mapa de luchas de poder. No existe una fórmula genérica que funcione en cualquier contexto.

2) Permitir que los sujetos sociales cobren un amplio margen de agencia en el proceso, en lugar de imponerles una cultura reíficada y prefabricada desde una posición externa. No existen unas características universales de una cultura definida a través de un etnónimo (marroquí o cualquier otro) que nos ayuden a saber lo que nos vamos a encontrar.

3) Partir de la base de que ellas y ellos son los que conocen y construyen su cultura, y son los únicos expertas y expertos en ella (Díaz de Rada, 2010). Propongo que se olviden los etnónimos (por ejemplo, marroquies) al planificar y desarrollar estas actividades. En su lugar, es más productivo dejar que las y los propios protagonistas decidan si quieren identificarse a través de ellos o de otro tipo de categorías de pertenencia, y cómo quieren hacerlo.

4) En cuanto a la técnica, más que una maestra que trae una coreografía ya elaborada, el agente de intervención debería ser un facilitador y un apoyo útil para las iniciativas que tengan los actores y actrices sociales. La base puede ser un género de baile que sea valorado por las protagonistas de ese contexto (lo cual no se puede saber sin consultarles previamente). A partir de ahí, lo fundamental es escuchar a sus cuerpos, más que escribir/prescribir sobre ellos. 


\section{Referencias bibliográficas}

Aixelá, Y. (2012). La presentación social del cuerpo marroquí en contextos migratorios. RDTP, 68(1), 19-48.

Bourdieu, P. (1987). Choses dites. París: Les Éditions de Minuit.

Browning, B. (1995). Samba: Resistance in Motion. Oxford: Westview Press.

Busto, B. (2012). El poder en el folklore: los cuerpos en NO-DO (1943-1948). Trans. Revista Transcultural de Música, 16, 1-30.

Carbonell i Paris, F. (1998). Desigualdad social, diversidad cultural y educación. En E. Aja, F. Carbonell, Colectivo Ioé, J. Funes e I.Vila (eds.), La inmigración extranjera en España. Los retos educativos. Barcelona: Fundación La Caixa.

Delgado, M. (1999). El animal público. Barcelona: Anagrama.

Díaz de Rada, A. (2010). Cultura, antropología y otras tonterías. Madrid: Trotta.

Díaz de Rada, A. y Jiménez Sedano, L. (2011). Variations on Diversity and the Risks of Bureaucratic Complicity. En L. Bradley y M. Pollock (eds.), A companion to the Anthropology of Education. Boston: Blackwell Publishing Ltd.

Foucault, M. (1986). Vigilar y castigar. Madrid: Siglo XXI.

García, A. (2009) La integración del alumnado inmigrante en el ámbito escolar: adecuar los recursos y aprovechar la diversidad. Cuadernos de Trabajo Social, 22, 297-314.

Gómez, L. (2009). Diccionario de Islam e islamismo. Madrid: Espasa.

Haenni, P. (2005). L'Islam de marché. Paris: Seuil.

Hall, E.T. y Hall, M.R. (2010). Los sonidos del silencio. En H. Velasco (comp.), Lecturas de Antropología Social y Cultural. Madrid: UNED

Hanna, J.L. (1988). Dance, Sex and Gender. Chicago: The University of Chicago Press.

Jiménez Sedanom L. (2012). El fantasma del racismo en las aulas: escuelas del más allá, niños que dan miedo y maestros cazafantasmas. En F.J. García Castaño y A. Olmos (eds.), Segregaciones y construcción de la diferencia en la escuela (pp.165-182). Madrid: Trotta.

Le Breton, D. (2007). El sabor del mundo. Buenos Aires: Nueva Visión.

Mernissi, F. (1992). El miedo a la modernidad. Madrid: Ediciones del Oriente y del Mediterráneo.

Mijares, L. (2006). Aprendiendo a ser marroquíes. Madrid: Edidiones del Oriente y del Mediterráneo.

Moreno González, A. Usán, S., Criado, C. y Santaforentina, A. (2013). Transitando identidades. La mediación artística en el proceso de rehabilitación de personas con problemas de adicciones. Cuadernos de Trabajo Social, 26(2), 445-454.

Quintero, A. (2009). Cuerpo y cultura. Madrid: Iberoamericana

Ramírez, A. (1998). Migraciones, género e Islam. Madrid: Agencia de Cooperación Internacional.

Ramírez, A. (2011). La trampa del velo. Madrid: Los Libros de la Catarata.

Sabbah, F. (1984). Women in the Muslim Unconscious. Londres: Pergamon Press.

Téllez, V. (2008 sept-dic,). La juventud musulmana de Madrid responde: lugar y participación social de las asociaciones socioculturales formadas o revitalizadas después de los atentados del 11-M. Revista de Estudios Internacionales Mediterráneos, 133-143.

Van Nieuwkerk, K. (2001). Changing Images and Shifting Identities: Female Performers in Egypt. En A. Dils y A. Cooper Albright (eds.), Moving History/ Dancing Cultures. Middletown, Connecticut: Wesleyan University Press.

Vázquez, O. (2001). Acción intercultural y trabajo social. Cuadernos de Trabajo Social, 14, 29-43.

Velasco, H. (1988). Creer es poder. RS Cuadernos de Relaciones Sociales, 31-32, 21-29.

Velasco, H. (2007). Cuerpo y espacio. Madrid: Ramón Aceres.

Williams, D. (2004). Anthropology and the Dance. Urbana and Chicago: University of Illinois Press.

Willis, P. (2008). Aprendiendo a trabajar. Madrid: Akal. 